\title{
Local Tilt Optimization of Photovoltaic Solar Panels for Maximum Radiation Absorption
}

\author{
Mauro Masili (iD) and Liliane Ventura \\ Department of Electrical Engineering, University of São Paulo (EESC/USP), Av. Trabalhador Saocarlense 400, São Carlos, \\ SP 13566-590, Brazil \\ Correspondence should be addressed to Liliane Ventura; lilianeventura@usp.br
}

Received 27 August 2019; Accepted 18 October 2019; Published 14 November 2019

Academic Editor: Yong Chen

Copyright (c) 2019 Mauro Masili and Liliane Ventura. This is an open access article distributed under the Creative Commons Attribution License, which permits unrestricted use, distribution, and reproduction in any medium, provided the original work is properly cited.

\begin{abstract}
Incident solar radiation on photovoltaic (PV) solar panels is not constant throughout the year. Besides dependence on the season, solar radiation is reliant on the location and weather conditions. For a given location on Earth, the best-fixed orientation of a PV panel can be determined by achieving the maximum incident solar irradiance throughout the year or for a predetermined period. In this paper, we use a sophisticated atmospheric radiative transfer model to calculate the direct and diffuse solar irradiation (radiant exposure) for the solar spectrum incident on PV solar panels to determine the best tilt angle of the panel in order to maximize absorption of solar radiation for selected periods. We used the Regula-Falsi numerical method to obtain the tilt angle at which the derivative of solar irradiation (concerning the tilt angle) approaches zero. Moreover, the spectral response of typical silicon cells is taken into account. These calculations were carried out in São Carlos (SP), a town in the southeast of Brazil. The best tilt angle was obtained for three selected periods. Additionally, we provide results for Southern latitudes ranging from $0^{\circ}$ to $-55^{\circ}$ in steps of $-5^{\circ}$ for the meteorological seasons. We have shown that for each period, there is an increase in solar radiation absorption compared to the traditional installation angle based exclusively on the local latitude. These calculations can be extended to any location.
\end{abstract}

\section{Introduction}

Electricity, as a type of energy, has become historically the cheapest and most widely used and has emerged as an essential and strategic resource for humanity. Nevertheless, electricity has not yet reached the entire world population, often depriving communities of the benefits this valuable resource can provide. In Brazil, there is a predominance of hydroelectric generation [1], comprising $64.54 \%$, while the photovoltaic (PV) generation is only $0.02 \%$. This alternative power generation is often presented as the only viable solution in remote regions or distributed forms of power generation and small installations.

Photovoltaic solar tracking systems aim to maximize exposure of the panels to sunlight keeping them as close as possible in the orthogonal position to the incident rays. However, in some situations such as in remote locations or rural areas, tracking systems may be impractical and/or expensive and therefore are not always applicable [2]. Thus, in many applications, fixed solar panels are the best option. Tracking systems may not fully take into account the incidence of radiation scattered by the atmosphere and the surroundings. Certainly, the direct normal irradiance from the sun is a major component of global irradiance incident on the panels. However, on cloudy days, solar panels still capture the sun's scattered radiation, indicating an important contribution. Figure 1 schematically shows the components of the total irradiance on a panel that is tilted at an angle $\beta$ to the horizontal.

There are various experimental and theoretical studies in the literature that determine ideal tilt angles of PV panels based on many assumptions to account for characteristics of the installation site [3-12]. An extensive literature review on the optimization of the tilt angle to maximize incident solar radiation has been provided elsewhere by Yadav and Chandel [13]. As pointed out by these authors [13], in most 


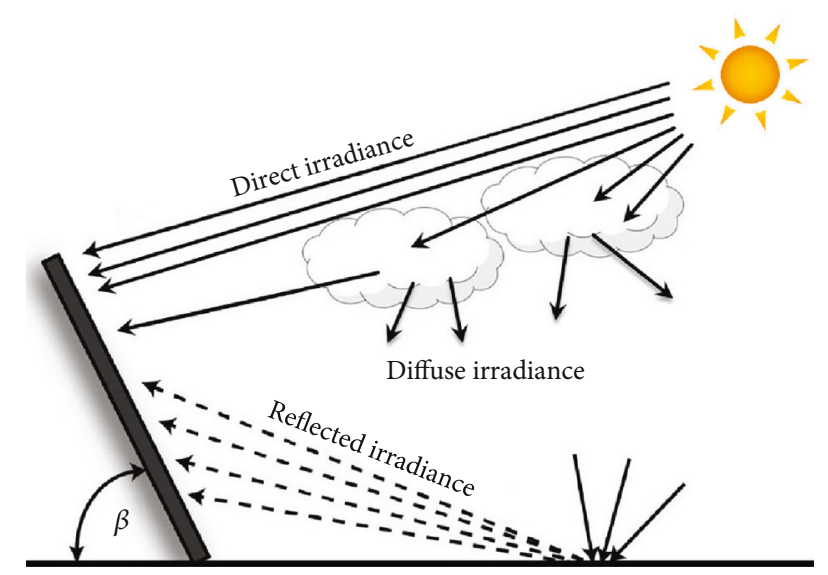

Figure 1: Solar irradiance and its direct, diffuse, and reflected components.

studies, direct and ground-reflected components of global irradiance were computed by using simplified models [13]. The diffuse component, by its nature, is more complicated and needs more sophisticated models [14-16]. Moreover, most studies in the literature [13] are for Northern hemisphere sites and use measured solar irradiation and/or use hybrid experimental/theoretical methods. Few works in the literature [13] rely exclusively on theoretical methods such as the present study. Thus, this research is useful for sites where measured data are unavailable or unreliable. To the best of the authors' knowledge, the literature is very scarce, if any, concerning the use of spectral responses of silicon cells in weighting solar irradiance to improve calculations. Indeed, none of the references cited in the aforementioned review [13] take into account the spectral responsivity of solar cells. Therefore, our work is situated in this area where there are limited available resources.

In view of this rationale, this paper is aimed at determining an optimum tilt angle for solar PV arrays in order to maximize incident solar irradiance on the array for specific periods, considering all solar irradiance components, i.e., direct normal, diffuse, and ground-reflected ones. In addition, the solar irradiance incident into the PV solar panel is weighted by the spectral response of typical silicon cells, strengthening the relevance of present work. We also use a very efficient and robust, yet simple, numerical method to determine the best tilt angle. This combination of methods is unique in the field. Furthermore, this is a strictly theoretical approach that does not rely on collected data, turning our approach universal.

\section{Methods}

In this section, the calculation and computer simulations will be detailed as follows.

2.1. Theoretical Foundations. A typical situation that this work aims to address is that of a flat solar panel, located in the Southern hemisphere, with a tilt angle $\beta$ to the horizontal and directed to the geographic North. In addition, the solar panel is exposed to atmospheric conditions described as clear

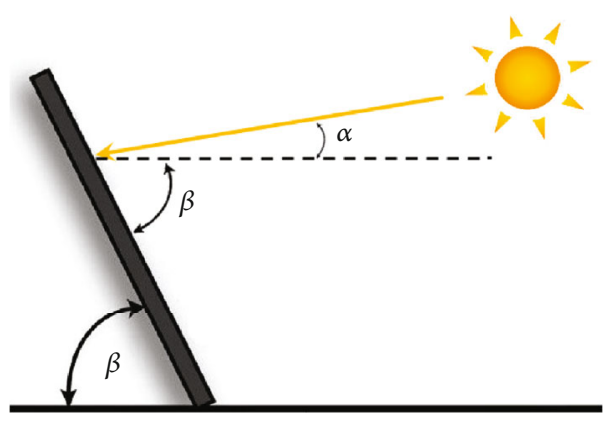

FIgURE 2: Angles involved in calculating solar irradiance on a tilted surface.

sky or without clouds. Thus, the calculation of spectral irradiance and solar irradiation at the tilted surface is required. For this purpose, the solar spectrum needs to be simulated using an atmospheric radiative transfer model [14-16].

The global solar irradiance $\left(\mathrm{W} / \mathrm{m}^{2}\right)$ for a given time and place is calculated in terms of the spectral irradiance:

$$
E(\mathbf{r}, t)=\int_{\lambda_{i}}^{\lambda_{f}} E(\lambda, \mathbf{r}, t) d \lambda
$$

In this expression, $E(\lambda, \mathbf{r}, t)$ is the solar spectral irradiance as a function of time and vector $\mathbf{r}$ represents the location's spatial coordinates, given by geographic coordinates (latitude, longitude, and altitude). In Equation (1) and onwards, $\lambda$ refers to the wavelength. Figure 2 shows the angles involved in the calculation of solar radiation on a tilted surface.

The direct normal irradiance, denoted by $E_{b}$, is obtained by:

$$
E_{b}(\mathbf{r}, t)=E(\mathbf{r}, t) \sin [\theta(\mathbf{r}, t)]
$$

in which the angular function $\theta(\mathbf{r}, t)=\alpha(\mathbf{r}, t)+\beta$ is the angle between the sun's incident rays and the panel's surface (see Figure 2) as a function of the location, the day of the year, and the selected time of day. The irradiance $E(\mathbf{r}, t)$ is given by Equation (1). The diffuse $\left(E_{d}\right)$ and reflected $\left(E_{r}\right)$ components are considered isotropic. To calculate the global solar irradiation $\left(\mathrm{J} / \mathrm{m}^{2}\right)$ on a tilted surface over a period, the three components of irradiance should be added, i.e.,

$$
H(\mathbf{r})=\int_{t_{i}}^{t_{f}}\left[E_{b}(\mathbf{r}, t)+E_{d}(\mathbf{r}, t)+E_{r}(\mathbf{r}, t)\right] d t .
$$

The integrals in Equations (1) and (3) are numerically calculated by a five-point Gauss-Legendre quadrature method. This scheme is very robust and provides accurate results by knowing only five points of the integrand. In order to increase precision, the integration intervals are subdivided and the integrals are calculated in each subinterval. The results of each subinterval are finally added up.

The challenge for these calculations is to obtain the spectral irradiance $E(\lambda, \mathbf{r}, t)$ at the panel surface in the desired 


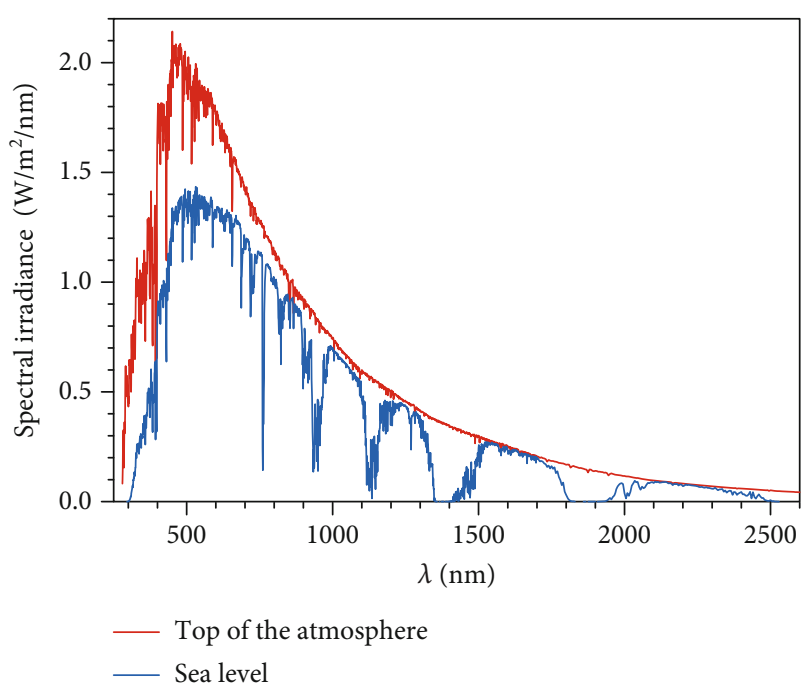

Figure 3: Simulated solar spectral irradiance at the top of the atmosphere (top curve) and at sea level (bottom curve) showing the attenuation due to gases and water vapor absorption bands in the atmosphere [14-16].

location for the day of the year and time of day. Furthermore, typical atmospheric data such as ozone concentration, water vapor column, and other information may be considered.

Figure 3 shows typical global solar spectral irradiance at the top of the atmosphere (top curve) and at sea level (bottom curve). Note the attenuation resulting from absorption bands of gases and water vapor at sea level.

Experimental data on solar irradiance are not available for all locations. Thus, irradiance must be obtained using an atmospheric model. Some solar spectrum modeling at the Earth's surface exists in the literature for semiquantitative applications $[17,18]$. In this paper, we propose using a well-known model in the literature, called the Simple Model of the Atmospheric Radiative Transfer of Sunshine, Version 2 (SMARTS2) [14-16]. This model uses solar spectral irradiance data collected by satellites as a primary data source. This model was chosen due to its accuracy, reliability, and availability of open-source codes, allowing it to be adapted to local conditions. This model proved to be precise and accurate when compared with proprietary and sophisticated models [19], as well as other methods in the literature $[20,21]$. The curves presented in Figure 3 were obtained using the SMARTS2 spectral radiation model [14-16].

Typically, PV panels absorb in the wavelength $(\lambda)$ ranging from $300 \mathrm{~nm}$ to $1200 \mathrm{~nm}$. Figure 4 shows the spectral response of a typical silicon solar cell [22]. The dots represent experimental data adapted from Ref. [22]. The solid line represents a polynomial adjustment as further explained in Section 3.2.

At wavelengths below $300 \mathrm{~nm}$ (ultraviolet), the glass absorbs most of the UV radiation and the electromagnetic response of the cells is very low. At intermediate wavelengths, the cell absorption increases with the wavelength and approaches the ideal performance. The response vanishes at long wavelengths (infrared) because silicon is a semiconductor with an indirect energy gap $E_{g}=1.12 \mathrm{eV}$ at room temper- ature [23], which is equivalent to a wavelength of $1107 \mathrm{~nm}$. Therefore, all calculations to be carried out will use, when needed, the absorption range of silicon cells, i.e., from $300 \mathrm{~nm}$ to $1200 \mathrm{~nm}$. Furthermore, the curve shown in Figure 4 should be used as a weighting factor of the spectral irradiance because the spectral response of a solar cell is not uniform for all wavelengths. Considering this, Equation (1) should be rewritten as follows:

$$
E(\mathbf{r}, t)=\int_{300 \mathrm{~nm}}^{1200 \mathrm{~nm}} E(\lambda, \mathbf{r}, t) S(\lambda) d \lambda,
$$

where $S(\lambda)$ is the spectral response of the photovoltaic cell, shown in Figure 4, i.e., $S(\lambda)$ acts as a weighting factor for the solar spectral irradiance $E(\lambda, \mathbf{r}, t)$. Because function $S(\lambda)$ is obtained experimentally, Equation (4) should be interpolated with the points presented in Figure 4 so that intermediate values are achieved in the integration. Thus, a high-order polynomial interpolation in multiple sectors should be used to ensure high accuracy with respect to the experimental points. Equation (4) can be related to the short-circuit current density $\left(J_{\text {SC }}\right)$.

In order to determine the best solar panel tilt angle to obtain the maximum power throughout the year, one could simply discretize and scan the tilt variable $\beta$, calculate the daily solar irradiation for each tilt angle for the entire year, and thus inspect the value of $\beta$ that provides the maximum annual solar irradiation. The main negative aspect of this approach is the unnecessary amount of calculations, overloading the computer resources. This approach can be avoided by adopting a more efficient procedure, which is determining the value of $\beta$ for which the derivative of the solar irradiation approaches zero. Therefore, one needs to calculate not only the solar irradiation given by Equation (3) but also its first derivative. In this study, a numerical fourth-order derivation method is used, i.e., the local truncation error is of the order of the step-size to the fourth power. This method uses Lagrange's formula for five-point derivation [24].

Once the derivative is calculated, its root can be sought. The root of a function can be found by various numerical methods, for instance, the bisection method, secant method, Newton-Raphson method, among others [25]. There are other powerful methods, such as Ridders' and Brent's methods [25], but they incur significant computational resources. Other algorithms, such as bisection and secant, are computationally simple and inexpensive, but may not always work [25]. The chosen method for this work is a compromise between speediness and robustness, i.e., the method is simple and numerically effective, reaching a solution with very few iterations. The method used is called Regula-Falsi [25], also known as the False Position method. This method has been used successfully in atomic [26-36] and solid-state physics $[37,38]$, in which the quantum mechanical equations call for very efficient numerical methods for minimizations. Thus, using this technique adds a novelty to the PV field. 


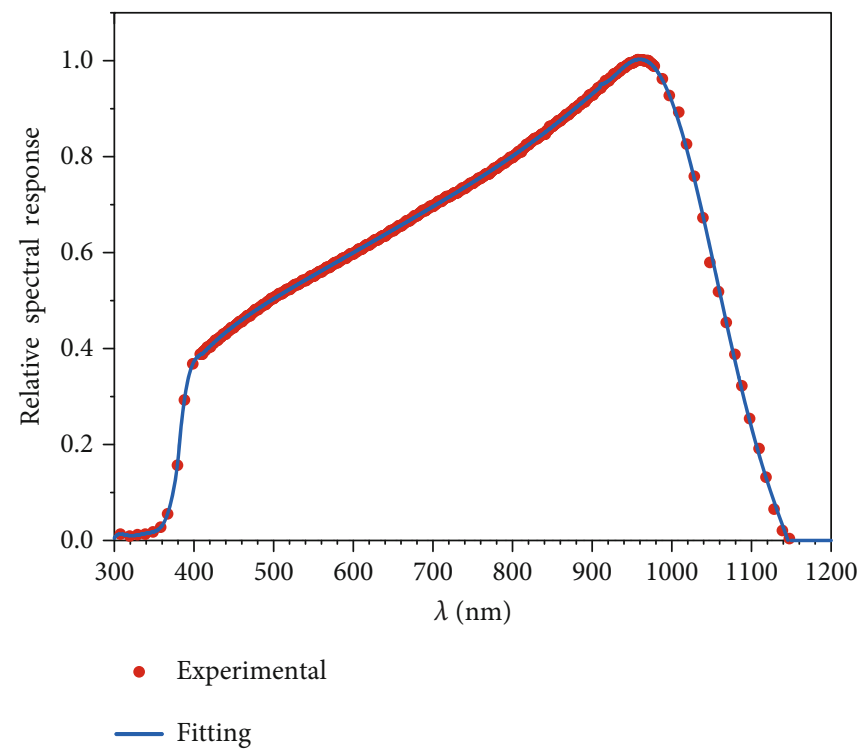

Figure 4: Typical spectral response of a silicon solar cell under glass. The dots represent experimental data adapted from Ref. [22]. The solid line is the polynomial fitting.

2.2. Computational Details. This work required intensive use of a computer system for floating-point operations in double precision $(\mathrm{REAL} * 8)$ aimed at minimizing rounding and propagation errors. The developed codes and free SMARTS2 [14-16] software that models the solar spectrum in the Earth's atmosphere use the FORTRAN programming language, which is still widely adopted in the scientific community, although nowadays, there are other programming languages that offer more flexibility. Nonetheless, the SMARTS2 [14-16] codes are only available in FORTRAN.

The SMARTS2 model requires input information about site location (geographical coordinates, altitude, azimuth, and tilt angle), date, and time. Furthermore, the model characterizes the local atmosphere (ozone concentration, aerosols, turbidity, and soil reflectance). Concerning the atmosphere, for Southern latitude calculations, we selected the SMARTS2 built-in "Tropical" standard atmosphere, which has typical gas concentrations without pollutants. In addition, the local environment was assumed as an urban area with a ground made of concrete. An assumption of a clear sky was made. Among a variety of output results, the data for spectral irradiances (direct, diffuse, and ground reflected) are returned to the main program to proceed with calculations.

\section{Results}

This section presents the results for solar irradiance and solar irradiation calculations from for the city of São Carlos (SP), Brazil, with geographic coordinates of latitude $-22^{\circ} 01^{\prime} 03^{\prime \prime} \mathrm{S}$, longitude $-47^{\circ} 53^{\prime} 27^{\prime \prime} \mathrm{W}$, and an average altitude of $854 \mathrm{~m}$. The calculations were carried out for all days of the year and, for each day, the incident radiation throughout the day was considered, i.e., from dawn to sunset. These results were used to determine the best tilt angle of a PV solar panel to optimize the absorption

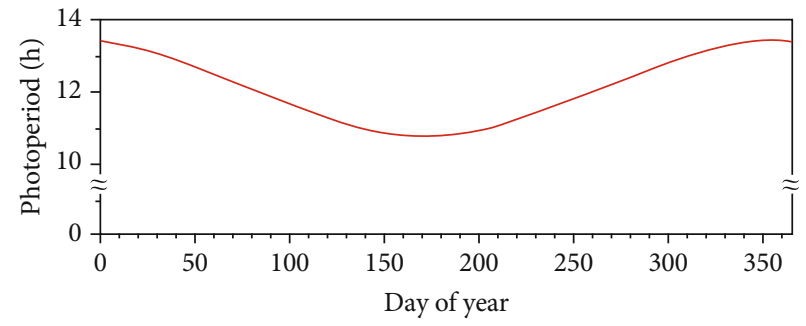

FIGURE 5: Solar day lengths throughout the year for São Carlos (SP), Brazil.

of solar radiation. This section is divided into subsections to present the results more clearly.

3.1. Photoperiod. Daylight length is called photoperiod and comprises the elapsed time from sunrise to sunset. Therefore, the sunrise and sunset times were calculated and the photoperiod was obtained for each day. The calculations of these times were made using the SMARTS2 [14-16] program by determining the time of day for which the sun elevation was zero, i.e., when the sun is on the horizon, more specifically, when half of the solar disk was below the horizon and the other half above. Figure 5 shows the photoperiod for each day of the year.

Figure 5 shows that daily sunlight lasts over 13 hours on summer days while it lasts slightly over 11 hours in the wintertime. The minimum period of sunlight extent occurs for day number 172 , which corresponds to June 21 . This day will be considered as half of the year and will be mentioned later as such.

3.2. Spectral Response. The first step was to fit the experimental points of the spectral response of a silicon solar cell, shown earlier in Figure 4. The fitting was done using 


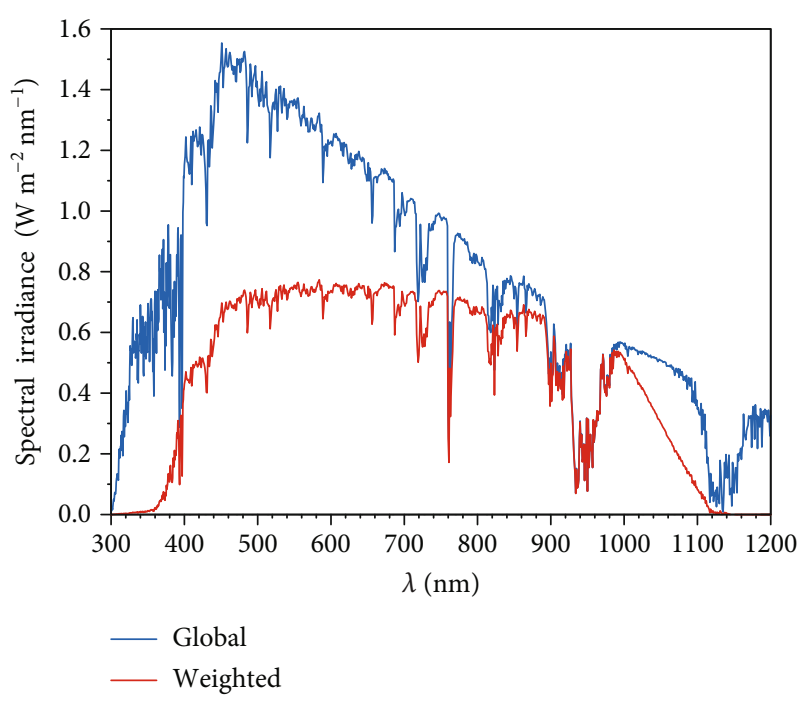

FIGURE 6: Solar spectral irradiance at a ground level calculated by the present atmospheric model. The top curve is the unweighted spectral irradiance and the bottom curve is the spectral irradiance weighted by the factor shown in Figure 4 .

polynomials up to the sixth order in four different spectral bands. These ranges are as follows (in $\mathrm{nm}$ ):

(1) $300 \leq \lambda \leq 380$

(2) $380 \leq \lambda \leq 410$

(3) $410 \leq \lambda \leq 920$

(4) $920 \leq \lambda \leq 1150$

These ranges were chosen to ensure minimum deviation in the fittings. For each of the above ranges, the fitting was of the sixth-order polynomial [25], each having, therefore, its own coefficients. Thus, the function obtained for the spectral response of the solar panel can be safely used instead of the experimental data, which are not available for all wavelength values. This polynomial $[S(\lambda)]$ was used to calculate the solar irradiance given by Equation (4).

3.3. Spectral Solar Irradiance. The spectral irradiance $E(\lambda, \mathbf{r}, t)$ was calculated using the SMARTS2 [14-16] codes for each value of $\mathbf{r}$ and $t$. An example can be seen in Figure 6, which shows the global solar irradiance (top curve) at ground level, in São Carlos. This curve can be compared with Figure 3 and it should be noted that the model accurately reproduces the solar spectrum observed at ground level.

The bottom curve in Figure 6 shows the global solar spectral irradiance weighted by the spectral response of the solar panel, i.e., this curve is the product of the solar irradiance by the cell's response for each wavelength. This weighted function should be integrated (compare Equation (4)) to give the total irradiance.

3.4. Total Solar Irradiance. The calculation of the spectral irradiance $E(\lambda, \mathbf{r}, t)$ allows one to calculate its integral to obtain the total solar irradiance, in accordance with Equation (4). The global solar irradiance consists of direct, diffuse, and reflected irradiances. For instance, for the $172^{\text {nd }}$ day of the year, which is June 21, the respective components of the global solar irradiances are shown in Figure 7. Note that, as expected, the main component is the direct irradiance on the solar panel. However, the diffuse and reflected components are not negligible. Collectively, they contribute about $20 \%$ of the overall irradiance.

The solar spectral irradiance weighted by the silicon cell's response was used for the total solar irradiance calculation (see Figure 6 (bottom curve)).

3.5. Solar Irradiation. This section presents the essential results of this study. The calculation of the solar irradiation (Equation (3)) for different inclinations of the solar panel shows the superficial energy density absorbed by the plate with the specified geometry. Calculations were made considering three scenarios that maximize solar irradiation (sometimes referred to as radiant exposure) into the panel depending on its tilt angle. The first scenario is the most general case in which the global solar irradiation throughout the year is considered, henceforth referred to as Period I. The second case (Period II) considers a six-month period centered in the day with less solar irradiation, i.e., June 21, which is day 172 of the year and its midpoint, as mentioned in section III A. Thus, the period considered for the calculation of this case spans three months before day 172 and three months after day 172 , ranging from day 80 to day 264, summing up 184 days between March 21 (day 80) to September 21 (day 264). This six-month period refers to the autumn and winter seasons in the Southern hemisphere. Finally, the third case (Period III) is concentrated in three months centered on day 172, i.e., from day 127 to day 217, corresponding to May 7 to August 5, respectively. These three scenarios are good choices to understand the dynamics of PV tilt angles throughout the year. Period I is the overall condition while Periods II and III are good in cases that are not connected with the grid (subdimensioned and/or with battery banks).

In order to generalize our approach, we provide results for Southern latitudes ranging from $0^{\circ}$ to $-55^{\circ}$ in steps of $-5^{\circ}$ considering four different periods. These are the standard meteorological seasons [39], which are more appropriate than the astronomical seasons (for this kind of calculation) because meteorological seasons are based on the annual temperature cycle.

As a first example, given a $30^{\circ}$ tilt for the solar panel, the solar irradiation for each day of the year can be seen in Figure 8. This figure shows the direct, diffuse, and reflected components, which are added together to obtain the global solar irradiation.

As expected, the solar irradiation is smaller in the central part of the graph, which corresponds to half of the year, namely, to the winter period (Southern hemisphere). The two maxima correspond to late summer and early spring. More precisely, they refer to the southern equinoxes. Figure 8 is a solar irradiation plot for each day of the year. Adding the values together for each day of the year, the annual superficial energy density is obtained. This amount is the total that the solar panel will be submitted during the year for the considered geometric condition. Thus, for a $30^{\circ}$ 


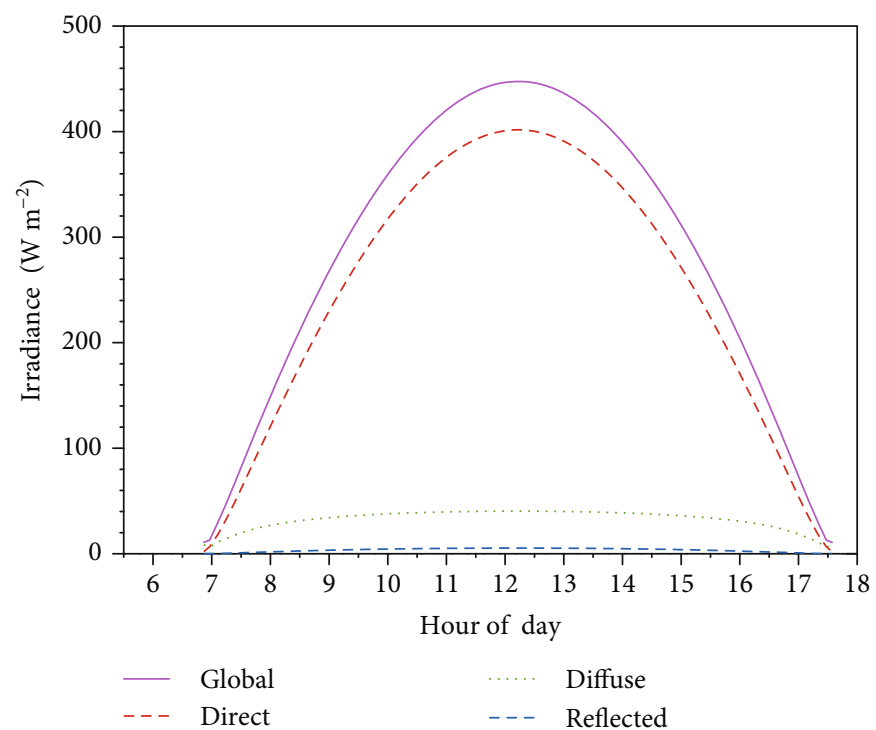

Figure 7: Weighted global solar irradiance and direct, diffuse, and reflected components for São Carlos, on June 21.

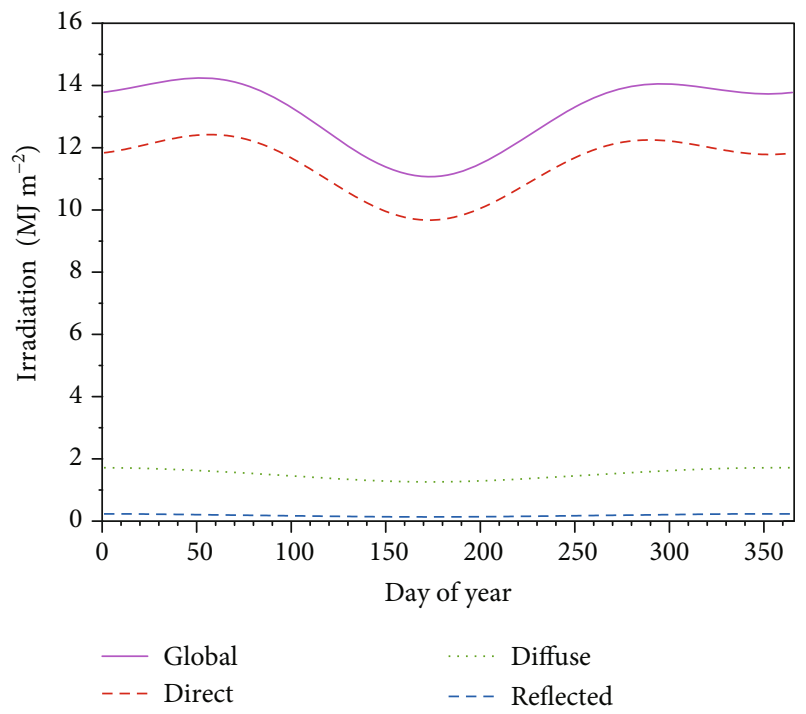

FIgURE 8: Annual solar irradiation incident on a solar panel with $30^{\circ}$ tilt, in São Carlos (SP), Brazil, at latitude $-22^{\circ} 01^{\prime} 03^{\prime \prime} \mathrm{S}$.

tilt of the PV panel, the total annual solar irradiation is $4758.1 \mathrm{MJ} / \mathrm{m}^{2}$. Recall that this value takes into account the spectral response of the PV panel, shown in Figure 4.

3.5.1. Maximization by Calculating the First Derivative. For all considered scenarios (Periods I to III for São Carlos and meteorological seasons for Southern latitudes ranging from $0^{\circ}$ to $-55^{\circ}$ ), the tilt angle of the solar panel is determined by the maximum solar irradiation within the period of each scenario.

The Regula-Falsi method for obtaining the root of the derivative of the solar irradiation has proven to be very numerically efficient because the root was obtained with an accuracy of eight decimal digits after only six iterations of
TABLE 1: Photovoltaic panel tilt angle to maximize the absorption of solar radiation at different periods of the year. The gain in solar irradiation compared to the standard angle is also shown.

\begin{tabular}{llcc}
\hline Period (day range) & Tilt $\left.^{\circ}{ }^{\circ}\right)$ & Solar irradiation $\left(\mathrm{MJ} / \mathrm{m}^{2}\right)$ & Gain (\%) \\
\hline I (1-365) & $23.22^{\circ}$ & 4783.14 & $0.02 \%$ \\
& $22.00^{\circ}$ & 4782.32 & \\
\hline \multirow{2}{*}{ II (80-264) } & $46.12^{\circ}$ & 2422.80 & $7.57 \%$ \\
\hline \multirow{2}{*}{ III (127-217) } & $22.00^{\circ}$ & 2252.16 & \\
& $53.92^{\circ}$ & 1177.56 & $14.19 \%$ \\
\hline
\end{tabular}

the algorithm. Evidently, there is no need for such precision in the tilt angle. One decimal digit is adequate for the physical installation of the panel. Convergence to one decimal digit would require only three iterations of the algorithm. Considering that the first two iterations are initial attempts required by the method, just one additional iteration would be sufficient. Therefore, the Regula-Falsi method proved to be extremely effective in determining the roots. The first two iterations are arbitrary depending on the desired range. In this paper, we have chosen $\beta=10^{\circ}$ and $\beta=50^{\circ}$ to start the iterations.

Table 1 shows the optimized tilt angle of a PV panel to maximize the absorption of solar radiation in the calculated periods.

Table 1 indicates the correct positioning for PV panels, for the town of São Carlos (SP) in Brazil, in order to obtain the maximum radiation absorption in the periods of the three considered scenarios. Note that the panel should always be directed to the geographic North and should not be determined by a compass. Table 1 also shows the total solar irradiation in each considered period and the gain in relation to the positioning of the panel at $22^{\circ}$, which is the default angle for São Carlos. It should be mentioned that this default angle is 


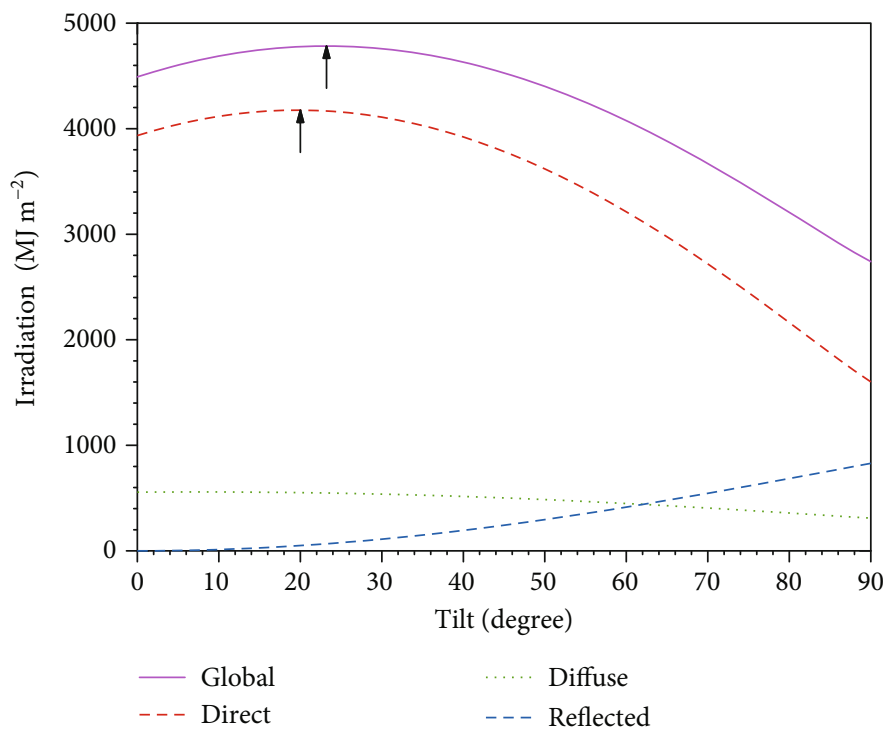

Figure 9: Components of solar irradiation incident on a solar PV panel as a function of inclination for Period I (year-long).

equal to the latitude installation site. Although the percentage gain for Period I may look small, we emphasize that usually PV panels are rarely tilted at $22^{\circ}$ (São Carlos) and not always directed to the North as they are placed on top of roofs that are not designed for this purpose. Indeed, roof inclinations in São Carlos are commonly designed in the $2^{\circ}-20^{\circ}$ range, depending on the roof covering.

3.5.2. Maximization by Direct Calculation. The calculation of maximization by direct calculation is redundant as the best tilt angle was determined in the previous section. However, the direct calculation is aimed at demonstrating the consistency of the results and ensuring that the searching method for the root of the derivative actually determines the point at which the total solar irradiation is maximum, i.e., its derivative with respect to the tilt angle of the solar panel is zero. The direct calculation is nothing more than calculating the annual solar irradiation to fixed intervals of the panel tilt angle. This allows the plot of the solar irradiation incident on the panel as a function of the tilt angle for the considered scenarios. Figure 9 shows the calculation for Period I as an example.

Note that the direct component reaches its maximum just before the global maximum, showing that the diffuse and reflected components have no negligible contributions in the overall calculation. To have a clearer understanding, Figure 10 shows the global solar irradiation incident in a solar panel as a function of inclination for the three considered periods.

The shift of the peak towards larger tilt angles is due to the maximum solar elevation of the days in the year that becomes smaller as the winter period approaches, causing the solar panel to be increasingly tilted in order to increase the radiation absorption.

3.5.3. Comparison and Generalization. It can be observed in Figure 10 that, indeed, the Regula-Falsi method was effective

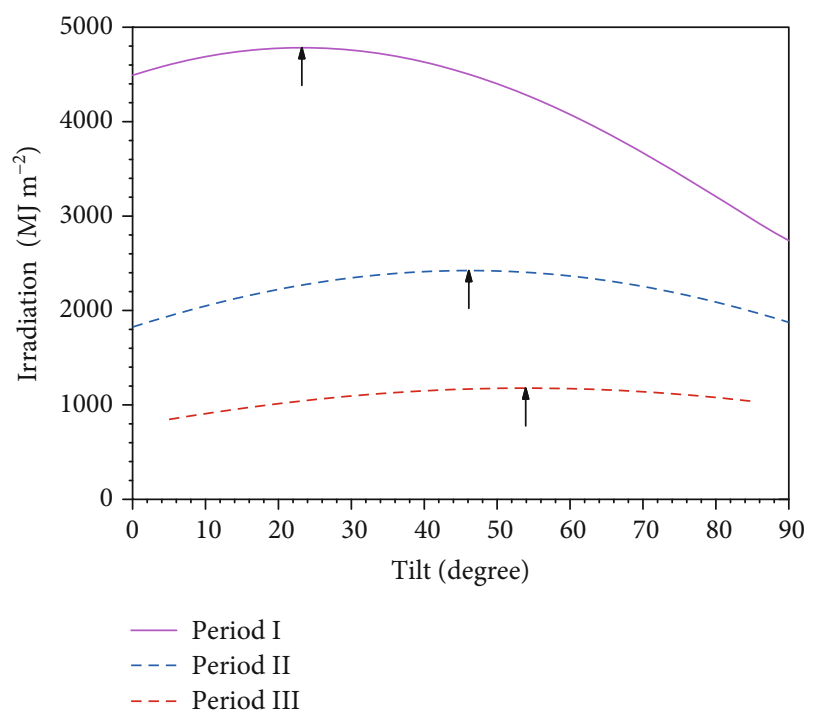

FIgURE 10: Solar irradiation incident in a PV solar panel as a function of tilt angle for the three considered periods.

in determining the tilt angle to maximize solar irradiation. Visual inspection also shows the accuracy of the method. Comparing the data in Table 1 with Figure 10, the consistency of the results can be observed, i.e., the values shown in Table 1 match the global maximum points of the respective curves.

Figure 11 shows the daily solar irradiation incident on the solar panel for each of the periods. For each period, the calculation was performed using the best solar irradiation panel tilt angle listed in Table 1, calculated using the proposed method. For shorter periods, the curves move upward, indicating that the solar irradiation is maximized as the tilt angle increases, and thus increases the absorption of solar radiation.

The curves in Figure 11 are the global solar irradiation, that is, the sum of the direct, diffuse, and reflected 


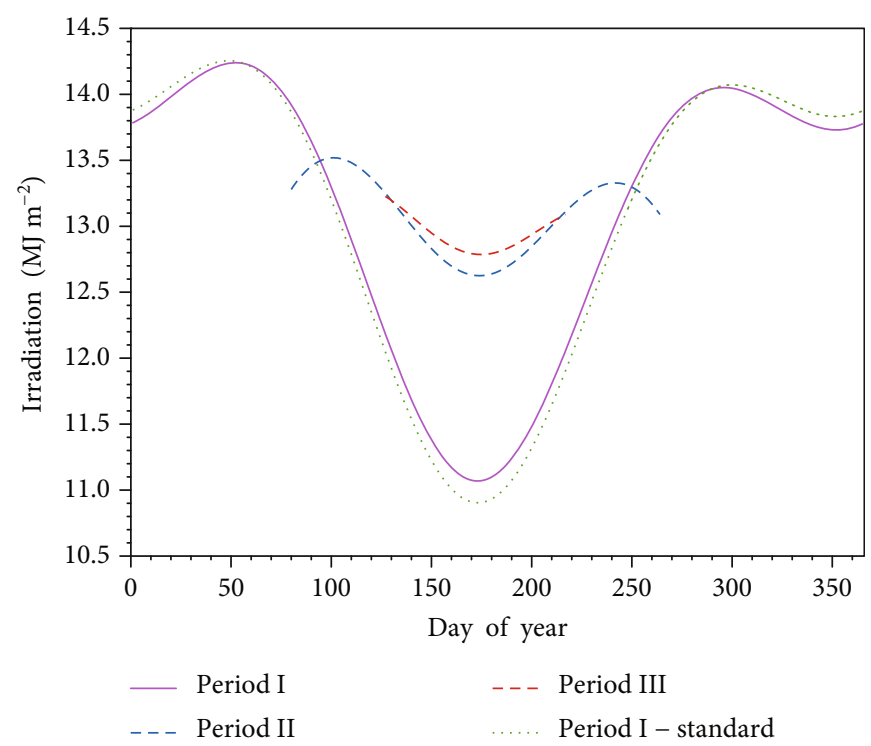

FIGURE 11: Global solar irradiation incident in a solar panel for tilt angles that maximize the absorption of radiation in the three considered periods. The irradiation for standard inclination for comparison purposes is also shown.

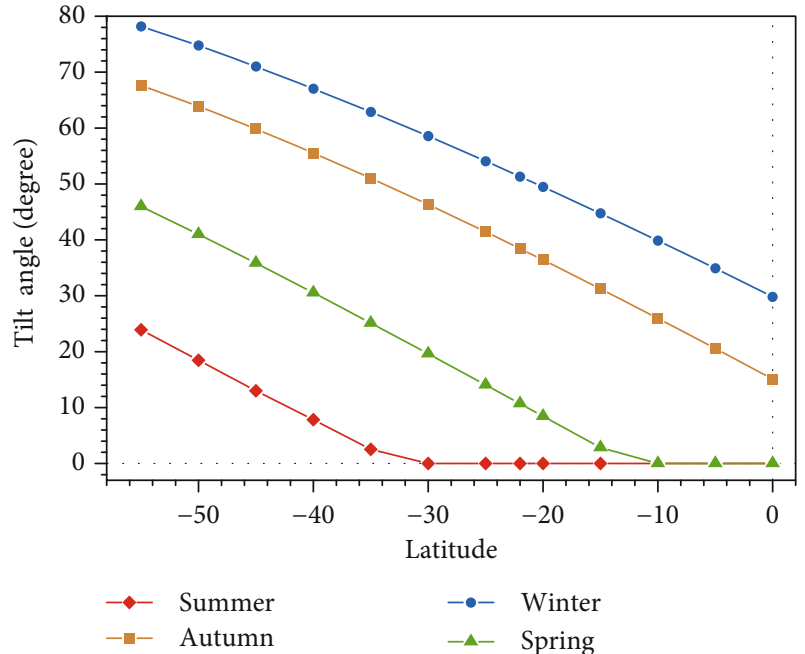

FIGURE 12: Calculated optimal tilt angle for meteorological seasons in Southern latitudes $\left(0^{\circ}\right.$ to $\left.-55^{\circ}\right)$.

components. Figure 11 also shows the global irradiation for standard inclination, i.e., the latitude tilt angle, for the sake of comparison.

In order to demonstrate the capabilities and versatility of this approach, Figure 12 shows results for Southern latitudes ranging from $0^{\circ}$ to $-55^{\circ}$ in steps of $-5^{\circ}$ for the meteorological seasons.

\section{Conclusions}

The irradiance and solar irradiation incident on PV panels were calculated, and the best panel tilt angle was determined from the maximum absorption of solar radiation in the chosen periods.
For irradiance and solar irradiation calculations, we proposed to use a diffusion and atmospheric radiative transfer model, which is well known and validated in the literature. Moreover, the solar irradiation is weighted by the response curve of the silicon PV panels. Subsequently, to determine the tilt angle of PV panels that retrieves the highest possible incident solar irradiation, we proposed the Regula-Falsi numerical method in order to find the point at which the derivative of the solar irradiation with respect to the tilt angle approaches zero. These three aspects are novelties to the field. This combination of methods is unique in the field. Furthermore, this is a strictly theoretical approach that does not rely on collected data, turning our approach universal.

As an application, the calculations were carried out for the city of São Carlos. The optimal tilt angle of a PV panel was obtained by the proposed method for three selected periods. Additionally, optimal tilt angles were provided for Southern latitudes and periods corresponding to the meteorological seasons.

For each considered period, this work showed an increase in solar radiation absorption compared to the general installation angle, which at best is equal to the latitude of the location. The highest gain was for Period III (winter season), which amounts to over $14 \%$ compared to the standard latitude tilt angle. At the level of $\mathrm{GJ} / \mathrm{m}^{2}$, this improvement is significant.

Finally, the computer codes developed are able to run autonomously, with minimum user intervention. The data set for each location of interest is provided to the main program as input files that can be edited by the user. Thus, these calculations can be extended to anywhere in the Southern or Northern hemispheres. Moreover, different desired periods of investigation are achievable. For instance, daily, weekly, or monthly values of optimal tilt angles can be provided for photovoltaic arrays. 


\section{Data Availability}

The data used to support the findings of this study are available from the corresponding author upon request.

\section{Conflicts of Interest}

The authors declare no conflict of interest.

\section{Authors' Contributions}

Aside from the SMARTS2 code, MM developed the other codes. In the remaining of this work, the authors have worked and contributed equally.

\section{Acknowledgments}

The authors are grateful to FAPESP (grant numbers 2013/08038-7 and 2014/16938-0), which financially supported our research. This study was financed in part by Coordenação de Aperfeiçoamento de Pessoal de Nível Superior-Brazil (CAPES) (Finance Code 001 (MM)).

\section{References}

[1] E. B. Pereira, F. R. Martins, A. R. Gonçalves et al., Atlas Brasileiro de Energia Solar, INPE, São José dos Campos, 2nd edition, 2017.

[2] N. Stefan, "Potentials for tracking photovoltaic systems and V-troughs in moderate climates," Solar Energy, vol. 45, no. 6, pp. 385-393, 1990.

[3] G. Lewis, "Optimum tilt of a solar collector," Solar \& Wind Technology, vol. 4, no. 3, pp. 407-410, 1987.

[4] A. Balouktsis, D. Tsanakas, and G. Vachtsevanos, "On the optimum tilt angle of a photovoltaic array," International Journal of Solar Energy, vol. 5, no. 3, pp. 153-169, 1987.

[5] K. Murat, S. Mehmet, B. Yunus, and D. Sedat, "Determining optimum tilt angles and orientations of photovoltaic panels in Sanliurfa, Turkey," Renewable Energy, vol. 29, no. 8, pp. 1265-1275, 2004.

[6] S. Naihong, K. Nobuhiro, K. Yasumitsu, and S. Hirotora, "Experimental and theoretical study on the optimal tilt angle of photovoltaic panels," Journal of Asian Architecture and Building Engineering, vol. 5, no. 2, pp. 399-405, 2006.

[7] B. Viorel, "Simple optimization procedure for silicon-based solar cell interconnection in a series-parallel PV module," Energy Conversion and Management, vol. 47, no. 9-10, pp. 1146-1158, 2006.

[8] C. Emanuelle, "Determining optimum tilt angles of photovoltaic panels at typical north-tropical latitudes," Journal of Renewable and Sustainable Energy, vol. 1, article 033104, 2009.

[9] Y. P. Chang, "Optimal design of discrete-value tilt angle of PV using sequential neural- network approximation and orthogonal array," Expert Systems with Applications, vol. 36, no. 3, pp. 6010-6018, 2009.

[10] J. Skerlić, J. Radulović, D. Nikolić, and M. Bojić, "Maximizing performances of variable tilt flat-plate solar collectors for Belgrade (Serbia)," Journal of Renewable and Sustainable Energy, vol. 5, no. 4, article 041820, 2013.

[11] I. S. Altarawneh, S. I. Rawadieh, M. S. Tarawneh, S. M. Alrowwad, and F. Rimawi, "Optimal tilt angle trajectory for maximizing solar energy potential in Ma'an area in Jordan," Journal of Renewable and Sustainable Energy, vol. 8, no. 3, article 033701, 2016.

[12] P. Sawicka-Chudy, M. Sibiński, M. Cholewa, and R. Pawełek, "Comparison of solar tracking and fixed-tilt photovoltaic modules in Lodz," Journal of Solar Energy Engineering, vol. 140, no. 2, article 024503, 2018.

[13] A. K. Yadav and S. S. Chandel, "Tilt angle optimization to maximize incident solar radiation: a review," Renewable and Sustainable Energy Reviews, vol. 23, pp. 503-513, 2013.

[14] C. A. Gueymard, "SMARTS2, a simple model of the atmospheric radiative transfer of sunshine: algorithms and performance assessment," Technical Report No. FSEC-PF-270-95, Florida Solar Energy Center, 1995.

[15] C. A. Gueymard, "Parameterized transmittance model for direct beam and circumsolar spectral irradiance," Solar Energy, vol. 71, no. 5, pp. 325-346, 2001.

[16] C. A. Gueymard, "The SMARTS spectral irradiance model after 25 years: new developments and validation of reference spectra," Solar Energy, vol. 187, pp. 233-253, 2019.

[17] H. L. Hoover and S. G. Marsaud, "Calculating solar ultraviolet irradiation of the human cornea and corresponding required sunglass lens transmittances," in Ophthalmic Optics, vol. 0601, pp. 140-145, Cannes, France, 1985.

[18] H. L. Hoover, "Solar ultraviolet irradiation of human cornea, lens, and retina: equations of ocular irradiation," Applied Optics, vol. 25, no. 3, pp. 359-368, 1986.

[19] P. Koepke, A. Bais, D. Balis et al., "Comparison of models used for UV index calculations," Photochemistry and Photobiology, vol. 67, no. 6, pp. 657-662, 1998.

[20] A. P. Souza and J. F. Escobedo, "Estimates of hourly diffuse radiation on tilted surfaces in southeast of Brazil," International Journal of Renewable Energy Research, vol. 3, pp. 207-221, 2013.

[21] D. Yang, "Solar radiation on inclined surfaces: corrections and benchmarks," Solar Energy, vol. 136, pp. 288-302, 2016.

[22] B. H. Hamadani and B. P. Dougherty, "Solar cell characterization," in Semiconductor Materials for Solar Photovoltaic Cells, M. Paranthaman, W. Wong-Ng, and R. Bhattacharya, Eds., vol. 218 of Springer Series in Materials Science, pp. 229-245, Springer, Cham, 2016.

[23] A. McEvoy, T. Markvart, and L. Castañer, Practical Handbook of Photovoltaics: Fundamentals and Applications, Elsevier, 2nd edition, 2012.

[24] M. Abramowitz and I. A. Stegun, Handbook of Mathematical Functions with Formulas, Graphs, and Mathematical Tables, United States Department of Commerce, National Bureau of Standards (NBS), Washington, 3rd edition, 1965.

[25] W. H. Press, S. A. Teukolsky, W. T. Vetterling, and B. P. Flannery, Numerical Recipes: The Art of Scientific Computing, Cambridge University Press, New York, NY, USA, 3rd edition, 2007.

[26] J. E. Hornos, S. W. MacDowell, and C. D. Caldwell, "Twoelectron wave functions in hyperspherical coordinates," Physical Review A, vol. 33, no. 4, pp. 2212-2224, 1986.

[27] M. Masili, J. E. Hornos, and J. J. De Groote, "Hyperspherical adiabatic approach for the helium atom," Physical Review A, vol. 52, no. 4, pp. 3362-3365, 1995.

[28] J. J. De Groote, M. Masili, and J. E. Hornos, "Highly excited states for the helium atom in the hyperspherical adiabatic 
approach," Journal of Physics B: Atomic, Molecular and Optical Physics, vol. 31, no. 21, pp. 4755-4764, 1998.

[29] J. J. De Groote, A. S. dos Santos, M. Masili, and J. E. Hornos, "Hyperspherical adiabatic approach for excitons bound to ionized donors in semiconductors," Physical Review B, vol. 58, no. 16, article 10383, 10388 pages, 1998.

[30] M. Masili, J. J. De Groote, and J. E. Hornos, "Nonadiabatic calculations of the oscillator strengths for the helium atom in the hyperspherical adiabatic approach," Journal of Physics B: Atomic, Molecular and Optical Physics, vol. 33, no. 14, pp. 2641-2652, 2000.

[31] J. J. De Groote, M. Masili, and J. E. Hornos, "Analytical functions for the calculation of hyperspherical potential curves of atomic systems," Physical Review A, vol. 62, article 032508, 2000.

[32] M. Masili and A. F. Starace, "Two-photon detachment cross sections and dynamic polarizability of $\mathrm{H}^{-}$using a variationally stable, coupled-channel hyperspherical approach," Physical Review A, vol. 62, no. 3, article 033403, 2000.

[33] M. Masili and A. F. Starace, "Static and dynamic dipole polarizability of the helium atom using wave functions involving logarithmic terms," Physical Review A, vol. 68, no. 1, article 012508, 2003.

[34] J. J. De Groote and M. Masili, "Low-lying doubly excited states of the helium isoelectronic series," Few-Body Systems, vol. 32, no. 4, pp. 249-266, 2003.

[35] M. Masili and J. J. De Groote, "Static and dynamic polarizabilities of $\mathrm{Na}^{-}$within a variationally stable coupled-channel hyperspherical method," Physical Review A, vol. 70, no. 5, article 054501, 2004.

[36] J. J. De Groote and M. Masili, "Electron affinity of the sodium atom within the coupled-channel hyperspherical approach," The Journal of Chemical Physics, vol. 120, no. 6, pp. 27672773, 2004.

[37] A. S. dos Santos, M. Masili, and J. J. De Groote, "Binding energies of excitons trapped by ionized donors in semiconductors," Physical Review B, vol. 64, no. 19, article 195210, 2001.

[38] J. J. De Groote, A. S. Santos, and M. Masili, "Dynamic polarizability of two-dimensionalD-ions in the presence of magnetic fields," Physical Review B, vol. 72, no. 3, article 033314, 2005.

[39] K. E. Trenberth, "What are the seasons?," Bulletin of the American Meteorological Society, vol. 64, no. 11, pp. 1276$1282,1983$. 

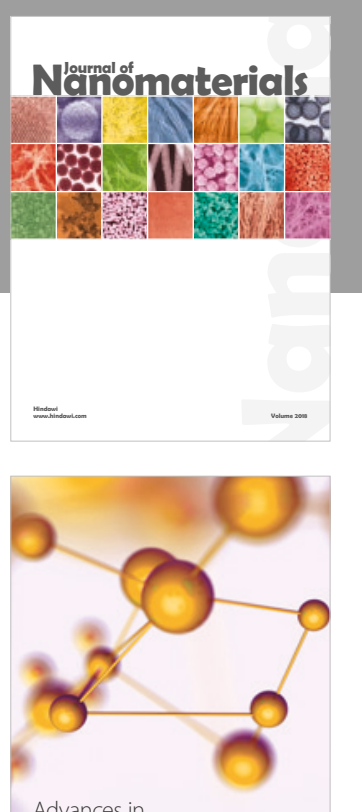

Physical Chemistry
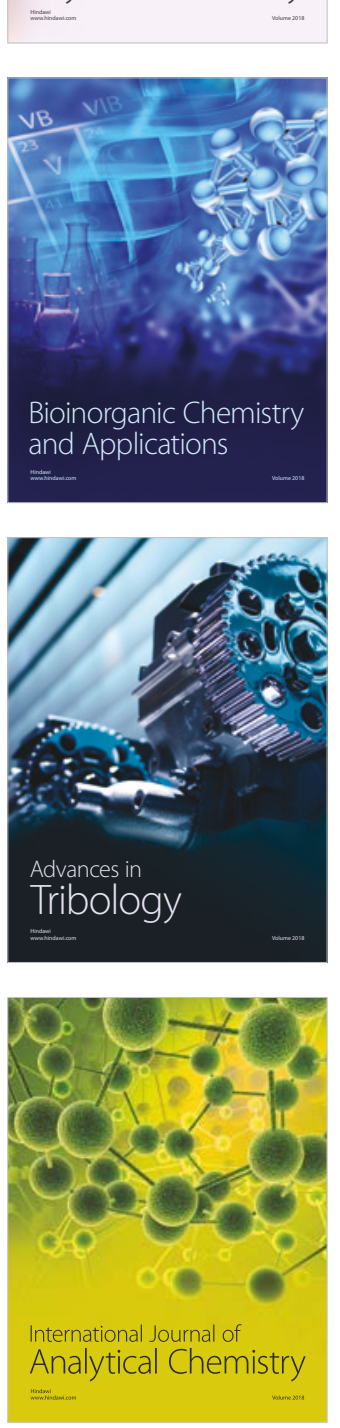

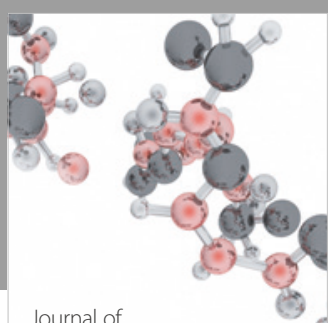

Analytical Methods

in Chemistry

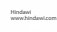

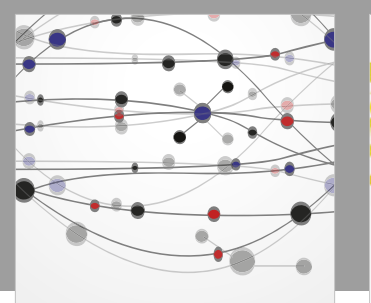

The Scientific World Journal

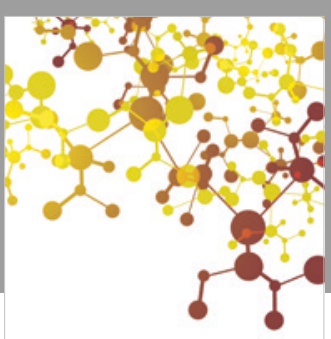

Journal of

Applied Chemistry
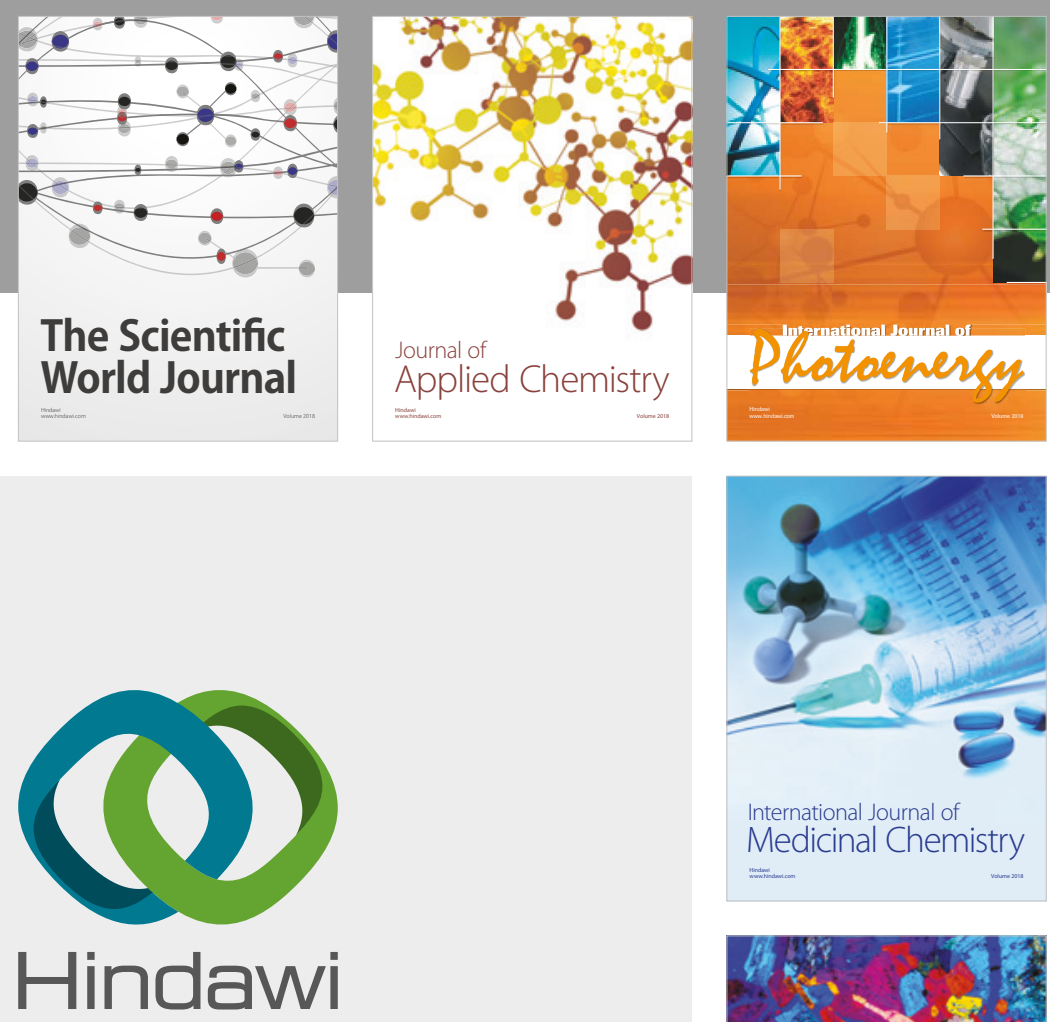

Submit your manuscripts at

www.hindawi.com
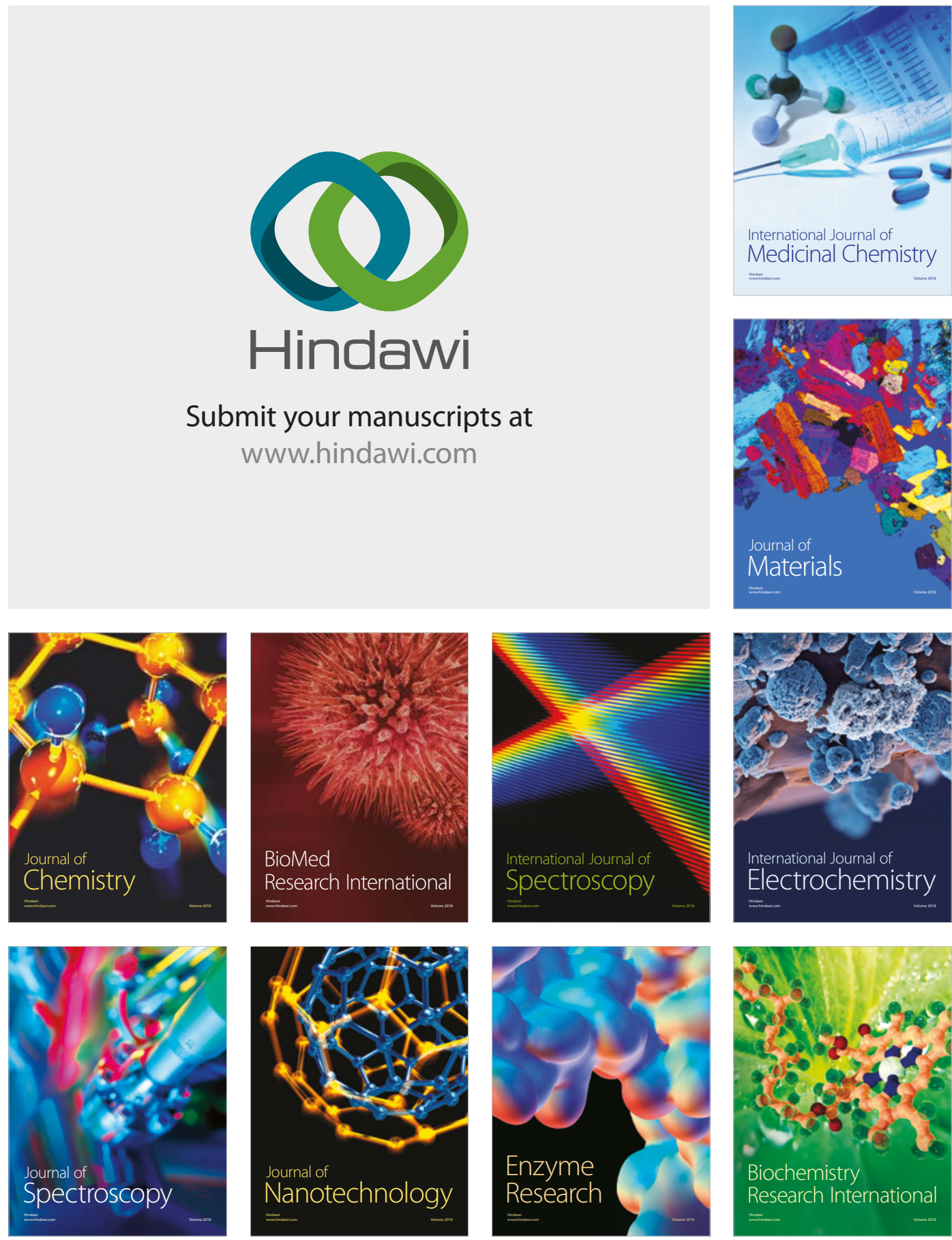
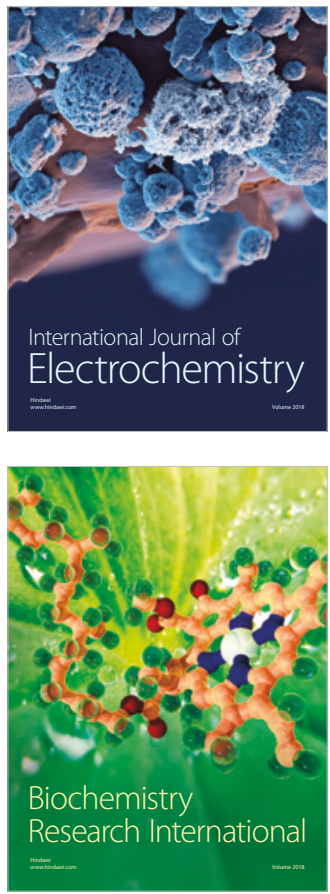